\title{
ARTICULACIÓN DE LA EDUCACIÓN MEDIA CON LA EDUCACIÓN SUPERIOR: INSTRUMENTO ADMINISTRATIVO PARA EL SECTOR EDUCATIVO DE LA CIUDAD DE IBAGUÉ
}

\author{
ARTICULATION OF THE BASIC EDUCATION WITH HIGHER EDUCATION: \\ ADMINISTRATIVE RESOURCE TO THE EDUCATION FOR PEOPLE IN IBAGUÉ
}

\author{
Gustavo Adolfo Rubio-Rodríguez*, Edgardo Mejia-Herrera** y Maud Eleonora Huertas-Valencia***
}

\begin{abstract}
RESUMEN
El presente estudio tiene por objeto determinar el proceso de articulación que se lleva a cabo entre la educación media y la educación superior en el Municipio de Ibagué; para ello, inicialmente se presenta la incidencia de la cobertura en educación al proceso de articulación educativa en Colombia, cómo soporte teórico al tema objeto de estudio. Seguidamente, se enseña la metodología utilizada en el proceso investigativo tomando como base la población sobre la cual se concentró el estudio (57 colegios públicos del municipio de lbagué). En este tamaño muestral se administraron encuestas, entrevistas y análisis de documentos, cuyos resultados permitieron concluir que existe un interés por parte de la Secretaría de Educación Municipal, de los colegios e instituciones técnicas y/o tecnológicas y de las mismas universidades para articularse. Sin embargo, no existe una orientación que encause unas políticas públicas claras para establecer cuáles son las competencias que deben desarrollar los estudiantes, y de esta manera alinear el conocimiento hacia el empoderamiento de actitudes que les permitan desarrollar las actividades requeridas por el sector productivo del país.
\end{abstract}

Palabras Clave: Articulación, educación media, educación superior.

\begin{abstract}
This study aims to determine the articulation process that takes place between secondary education and higher education in the city of Ibague; to do this, initially the incidence of coverage is education to the process of joint education in Colombia, how theoretical support to the subject under study. Next, the methodology used in the research process based on the population on which the study ( 57 public schools in the municipality of lbague) appears concentrated. In this sample size surveys, interviews and document analysis were administered, the results led to the conclusion that there is an interest from the Municipal Department of Education, schools and technical and/or technological institutions and universities themselves to articulate.
\end{abstract}

Fecha de recepción: Mayo 04 de 2015 / Fecha de aceptación: Junio 18 de 2015

Tipología: Artículo de Investigación Científica y Tecnológica

Para citar este artículo: Rubio, R. G., Mejía, H. E. \& Huertas, V. M. (2015). Articulación de la educación media con la educación superior: instrumento administrativo para el sector educativo de la ciudad de Ibagué. Praxis. Vol. 11, 76 - 88

*(c) Ph.D. Universidad Cooperativa de Colombia. Colombia. Email: gustavo.rubio@campusucc.edu.co

**Magister. Universidad Cooperativa de Colombia. Colombia. Email: edgardo.mejia@campusucc.edu.co

***Magister. Universidad Cooperativa de Colombia. Colombia. Email: maud.huertas@campusucc.edu.co 
However, there is no clear guidance encause public policies to establish what competencies they should develop students are, and thus align the knowledge to empower attitudes to develop the activities required by the productive sector of the country.

Keywords: Articulation, secondary education, higher education.

\section{INTRODUCCIÓN}

$\mathrm{P}$ rimeramente, es necesario ubicar dentro del contexto educativo el verdadero concepto de la articulación media con la superior. Según el documento elaborado por el Ministerio de Educación Nacional y el Servicio Nacional de Aprendizaje SENA (2012) afirman que:

La articulación es un proceso pedagógico y de gestión concertado que favorece el acceso, permanencia y movilidad de los estudiantes entre la educación media y otros niveles y ofertas educativas; este proceso genera el reconocimiento de los aprendizajes obtenidos en distintos escenarios formativos y el mejoramiento continuo de la pertinencia y calidad de la educación media, la educación superior, la formación profesional integral y la educación para el trabajo y el desarrollo humano. (p.7)

Otros autores como Olga Beatriz Cordero de Barrientos (2003, citado en Guerrero-Mosquera, Martínez-Benavides, \& Guazmayán-Ruiz 2012) sostienen que:

La articulación educativa es un modelo teórico-práctico de organización y gestión, aplicable a los distintos niveles y campos del sistema educativo, dinámico, flexible, abierto y complejo; que se construye y sostiene en procesos de interacción sistémica, entre todos los actores educativos, para establecer relaciones funcionales y significativas entre los componentes del campo de la educación, curriculares y metacurriculares, a fin de lograr metas consensuadas de la enseñanza. (p.745)

La articulación de la educación en Colombia nace como una propuesta del Gobierno Nacional en cumplimiento de las orientaciones del proyecto principal de educación para América Latina y el Caribe (PROMEDLAC, 1996), liderado por la UNESCO; éste proyecto tiene como fin lograr la continuidad entre el estudio de la educación media y el de la educación superior, así como lograr la preparación para el estudio hacia el trabajo, con el propósito de cualificar la mano de obra y ampliar la cobertura en la educación superior, aumentando el número de jóvenes colombianos en la educación técnica, tecnológica y superior con la finalidad de resolver parcialmente la problemática del desempleo, con competencias para la inserción en el mundo laboral, y propendiendo por un desarrollo sostenible de las economías regionales.

Para profundizar sobre la articulación en la educación, se deben conocer las condiciones fundamentales de los niveles de educación media, entendiéndose como tal los niveles de bachillerato que comprende del décimo grado hasta el grado once, así como los niveles técnico profesional, tecnológico y superior basados en la cobertura de educación existente y en las competencias que se vienen impartiendo, con el fin de brindar una educación de calidad acorde con las necesidades del mercado laboral. Sobre las competencias que se deben abordar en el proceso de articulación, el Ministerio de Educación Nacional (2008) sostiene lo siguiente:

Incluir las competencias en los estudiantes constituye uno de los elementos básicos para mejorar la educación; por tanto, es un esfuerzo que debe quedar consignado en el plan de mejoramiento institucional. Se requiere de un enfoque que dé paso a una educación más integradora, que articule la teoría y la práctica y que garantice aprendizajes aplicables a la vida cotidiana. (p.5)

En Colombia se ha legislado con el fin de cumplir con el objeto de articular la educación en un marco legal que advierte la Constitución 
Nacional, sin embargo, este esfuerzo no ha sido suficiente para que se haya dado una verdadera articulación entre la educación media con la educación superior y el mundo laboral. Esta afirmación se puede soportar en el marco de la ponencia presentada por el profesor Andrés Díaz, cuando señala:

He escuchado muchas veces la necesidad de que alguien se dedique a organizar, por ejemplo, las propuestas sobre articulación entre educación media y superior, pero lo que hay que hacer es una organización de toda la legislación educativa, que lo que existe quede claro y que lo que se deba agregar tenga un adecuado o coherente proceso de integración -para que no pase lo que he vivido en las regiones donde padres, profesores y administrativos hablan en tres idiomas distintos, con distintas expectativas y desde distintas normas sobre un deber ser de la educación. (Díaz, 2012, p.30)

Para el caso concreto, en la ciudad tomada como unidad de análisis geográfica (Ibagué), se ha venido impulsando a través de la secretaría de educación, la articulación de la educación media con la educación superior, con el ánimo de focalizar esfuerzos que permitan mejorar la calidad educativa, preparar a los estudiantes para la vida productiva y contribuir al mejoramiento del empleo. Dada esta iniciativa, la construcción de este manuscrito se adelanta con el fin de conocer y evaluar el estado en el que se encuentra el proceso de articulación educativa del municipio de Ibagué, con el propósito, a su vez, de lograr generar alternativas de solución al flagelo de descomposición social y desempleo que afronta la ciudad, como también de visualizar y encaminar las acciones concurrentes al proyecto nacional.

Este documento tiene implicaciones prácticas y de gran relevancia, en atención al manifiesto interés evidenciado por parte de las comunidades educativas de la ciudad de Ibagué, en hacer un seguimiento y una evaluación de la articulación de la educación, ya que de este tipo de procesos dependerán en buena medida las políticas y estrategias que, en materia educativa, se implementarán en el futuro.
No se puede dejar de un lado que la crisis social de la sociedad latinoamericana, incluida naturalmente la colombiana en sus extensiones de vulnerabilidad, ha generado un ambiente de inseguridad que pone en riesgo el normal funcionamiento de los países, como también el comportamiento y la subsistencia de las instituciones educativas del nivel superior (Vela, 2000). En este sentido, los estados deben ser asiduos vigilantes en toda acción que se promulgue en materia educativa, a fin de mejorar la funcionalidad de sus estamentos, y la construcción de políticas públicas que permitan el nexo entre la educación media con la educación superior.

\section{Antecedentes de la articulación de la educa- ción media con la superior}

El marcado crecimiento de los procesos en la educación superior en América Latina durante las últimas décadas, el cual va acompañado de problemas de calidad y pertinencia, se genera como producto de la amplia masificación de la educación superior presentada en todo el mundo (Schzuartzmann, 2001). Para Colombia, los lineamientos establecidos para la educación, específicamente para la articulación, no son aislados y corresponden a las tendencias de la educación en los países del mundo y en particular a los de América Latina y el Caribe, los cuales fueron concertados en los diferentes PROMEDLAC organizados por la UNESCO. El origen de esta iniciativa tiene como propósito crear un horizonte de competencia, calidad y articulación de la educación para un mundo globalizado y competitivo.

Para ubicar el tema objeto de estudio, resulta conveniente destacar en el contexto internacional la forma como se ha concebido este proceso. En México por ejemplo, las familias y las fuerzas influyentes cohesionan a las autoridades municipales por la apertura de instituciones educativas media-superior (Tapia, 2012, citado en Weiss, 2012). Esta estrategia es válida, dentro del afán que les asiste a los padres de familia por preparar a sus hijos para el mundo laboral; no obstante, si bien dicha estrategia se convierte en un bien 
apreciado, no siempre surtirá el efecto esperado, en razón de la interrupción que se presenta entre el paso de la educación media a la educación superior.

Para el caso de Uruguay, este país ha sido pionero en procesos de articulación tal y como lo evidencia "la creación de bachilleratos tecnológicos, el impulso de carreras técnicas y tecnológicas en la Universidad del Trabajo del Uruguay, la cual brinda estudios de educación media técnica y una carrera superior de Ingeniería Tecnológica” (Camargo, Garzón \& Urrego, 2012). Asimismo, se debe acotar que para el ingreso de un estudiante a una Universidad Uruguaya, un condicionamiento indiscutible es haber adelantado el bachillerato en una de las instituciones de bachillerato tecnológicos destinadas para tal fin (Camargo, et al., 2012).

También vinculante es mencionar, que en el contexto latinoamericano la articulación educativa no es un tema de actualidad; ésta ha sido ideada para optimar la educación, con la intención de gestar una evolución en esta materia en cada región. "Países como Venezuela, Colombia, Ecuador, Perú y Bolivia abordaron la articulación de la educación agropecuaria y lo rural” (IICA \& UNESCO, 2006, citado en Guerrero-Mosquera, et al., 2012).

De todo lo anterior, se puede inferir y a la vez complementar que la comunidad estudiantil de América Latina, ha afrontado y enfrenta serios problemas de adaptabilidad al ingresar a su primer año de Universidad (Uzuriaga, Posso \& Martínez 2013); por tanto, se debe propender por formular verdaderas soluciones que permitan que los estudiantes inicien y terminen sus estudios sin ningún contratiempo. Para esto, hay que tener en cuenta que cada país tiene retos muy diferentes en materia educacional, sin embargo, para neutralizarlos deben concurrir aspectos como "el desarrollo de currículos flexibles orientados al desarrollo de habilidades fundamentales más transversales, tales como la adaptación, la capacidad de resolver problemas y las habilidades de comunicación, así como a acciones de orientación vocacional" (Banco Mundial, 2007, citado en Díaz 2012).

\section{Incidencia de la cobertura en educación al proceso de articulación educativa en Colombia}

Antes de esgrimir argumentos y concentrar esfuerzos por articular procesos educativos, es necesario repensar acerca de la calidad que ostenta la educación en Colombia y también de la cobertura que alcanza la misma. El gobierno Colombiano se ve abocado a implementar políticas públicas en materia educativa con las cuales alcance, verdaderamente, mayor cobertura y mejor calidad en la educación; la mal llamada revolución educativa, exteriorizada por diferentes gobernantes no ha tenido nada de revolución; han consistido en planes fallidos de cobertura sin inversión adicional (Vasco, 2006).

Para efectos de cobertura, Colombia presenta una de las tasas más bajas de escolarización en la educación superior en américa latina. "El rápido crecimiento de la matrícula de educación superior a partir de 1960 no logró reducir la brecha existente con respecto a países como Argentina, Chile, Costa Rica, Uruguay o Venezuela" (Misas, 2004, p.54). La hegemonía neoliberal no ha logrado globalizar la educación dentro del contexto nacional; esta versión de la globalización se encuentra muy distante de la versión neoliberal, la cual presenta un discurso sobre el progreso con el objeto de revitalizar formulas económicas, y en consecuencia formulas acerca de cómo renovar la educación, la política y la cultura (Burbules \& Torres, 2001).

La cobertura educativa en Colombia deriva de un proceso de la articulación entre la formación media y la formación superior. Dicho proceso ha contribuido notablemente a reducir los índices de carencia económica de la población, producto de la empleabilidad la cual genera mayor competitividad para las regiones. Asimismo, permite a su vez a cerrar fisuras de inequidad en la educación, a estimular la permanencia de los jóvenes en las regiones y a la formación de ciudadanos 
competentes que concurren al desarrollo y al bienestar de las mismas (Documento de trabajo MEN-SENA, 2012).

El Gobierno colombiano a través del Ministerio de Educación, con el fin de conseguir mayor ampliación de cobertura, se planteó mejorar la retención de estudiantes en el tránsito de la educación media con la educación superior. El Ministerio, para el año 2012 se encontraba desarrollando el proyecto denominado "competencias laborales, formación para el trabajo y pertinencia en la educación media”, el cual consistía en acompañar a las secretarías de educación de todo el país, en la formulación e implementación de planes de formación en competencias laborales para las instituciones educativas y su articulación con el mundo productivo. No obstante, en la actualidad se tendría que revisar si la educación técnica, media y superior se están ofertando articuladamente en correspondencia con las demandas de la dinámica productiva de la regiones (MEN, 2012).

Una de las dificultades que afronta el país en materia educativa, la cual se concentra primordialmente en las instituciones técnicas y tecnológicas, consiste en el ofrecimiento de programas de baja calidad y pertinencia, debido a los problemas económicos que caracterizan en gran número a este tipo de instituciones (Camargo, et al., 2012). Sin embargo, en medio de esta debilidad, la articulación emerge como alternativa pedagógica y de gestión concertada, y a su vez se convierte en el mecanismo que permite el ingreso y la estabilidad de los estudiantes, como también la movilidad de éstos entre la formación media y demás ofertas educativas de orden superior (MEN, 2012). Asimismo, la articulación según el MEN contribuye a: “ofrecer una educación de calidad, responder a las necesidades del sector productivo (pertinencia), aumentar la permanencia en el sistema (cobertura), construir un país más justo y equitativo” (Documento de trabajo MEN, 2009:19).

\section{METODOLOGÍA}

El presente trabajo se adelantó bajo un enfoque cuali-cuantitativo, tratando de analizar diferentes comportamientos al interior de las unidades de análisis que formaron parte de este proceso investigativo, las cuales recayeron en el objeto concreto que se investigó (rectores, docentes y padres de familias de diferentes instituciones educativas de la ciudad de Ibagué). Bajo esta concepción, fue necesario indagar a través de las entrevistas y las encuetas la representación que caracteriza la población objeto de estudio, a fin de conocer sus diferentes opiniones. Dichas entrevistas y encuestas están significadas en un cuestionario cuyos resultados, luego de su administración, logró reflejar los resultados esperados en cada uno de los objetivos específicos, para así facilitar el cumplimiento del propósito general.

Es necesario en este apartado, hacer énfasis en la importancia que representa el cuestionario y su constructo para la presente investigación dentro del enfoque que se ha decidido adoptar, toda vez que muchos de los metodólogos coinciden en afirmar que un cuestionario es una técnica que permite recolectar información con fines de tomar decisiones, dentro de la búsqueda de alternativas para la solución de problemas. Para Hernández, Fernández y Baptista (2010) “el cuestionario consiste en un conjunto de preguntas respecto de una o más variables” (p.217).

La población objeto de investigación se concentró en 57 colegios públicos del municipio de Ibagué, en donde se indagaron a los siguientes actores: rectores, estudiantes, profesores, padres de familia de la educación media y de los colegios públicos articulados en el municipio de Ibagué, Tolima (Colombia). Asimismo, se recolectó información facilitada por parte de los directivos de la Secretaría de Educación Municipal, el Servicio Nacional de Aprendizaje (SENA) y la Corporación Unificada Nacional de Educación Superior (CUN).

La muestra se seleccionó utilizando el muestreo aleatorio estratificado con afijación proporcional; de acuerdo a la población existente se obtuvo un tamaño mínimo de muestra de 333 estudiantes, distribuidos por estrato. Para el caso de los demás actores ubicados en las instituciones educativas como: rectores, docentes y padres de familia, se trabajó exhaustivamente, al seleccionar las personas que en su totalidad estaban involucradas en el proceso de articulación, tomando muestras 
de documentos que así lo demostraran. Para el caso de la Secretaría de Educación Municipal de Ibagué, el SENA y la CUN, se procedió a recoger la información mediante el método de entrevista focal a los representantes de cada uno de estos organismos educativos.

\section{RESULTADOS}

A continuación se enseñan las gráficas con sus respectivas interpretaciones, correspondientes a las categorías más relevantes del proceso investigativo, las cuales dan lugar a las conclusiones emitidas por parte del grupo investigador:

\section{Categoría: Pretensión del proceso de articulación}

Las preguntas que comprende la figura número 1 se engloban en el siguiente cuestionamiento: ¿que se busca con el proceso de articulación?; en este, se pretende identificar si los actores involucrados en el proceso de articulación, conocen de la intención de esta política educativa. Existe una respuesta favorable por parte de los rectores y docentes, quienes en un $37,5 \%$ y un $46,2 \%$, respectivamente, consideran que la articulación busca vincular el sistema productivo con el sistema educativo y con el mundo del trabajo. En el caso de los estudiantes por el contrario, se presenta una polarización en cuanto a la intención de este proceso. De otro lado, los padres de familia se inclinan a que, con la articulación se facilita el ingreso de los estudiantes de educación básica a la educación superior.

\section{Figura 1}

Pretensión del proceso de articulación

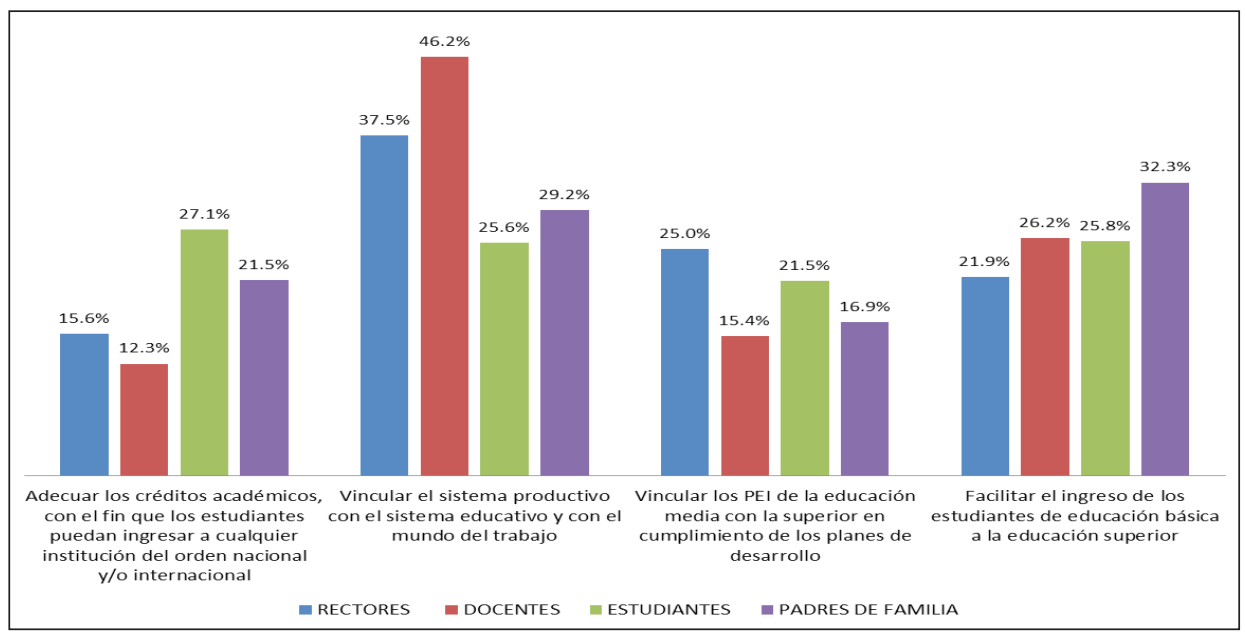

Fuente: elaboración propia. 
Según el Ministerio de Educación Nacional (2010) "la articulación es un proceso pedagógico y de gestión que implica acciones conjuntas para facilitar el tránsito y la movilidad de las personas entre los distintos niveles de formación y los sectores productivos (...)" (p.6). Por lo anterior, las instituciones de educación técnica y tecnológica, las instituciones de educación media y educación superior, deben propender fundamentalmente por vincular el sector productivo con el sistema educativo y con el mundo del trabajo.

\section{Categoría: Instituciones con las cuales se viene adelantando el proceso de articulación}

En la figura número 2 se observa que el Servicio Nacional de Aprendizaje (SENA), es la entidad que mayor articulación presenta con las instituciones de educación media en el municipio de Ibagué; continúan en ese orden la Universidad del Tolima, la Universidad Cooperativa de Colombia, la Corporación Unificada Nacional, el Instituto Técnico Tolimense de Formación Profesional, el Centro Técnico San José, el cual es una extensión de la Universidad de Ibagué, y la Corporación John F. Kennedy.

\section{Figura 2}

Instituciones con las cuales se viene adelantando el proceso de articulación

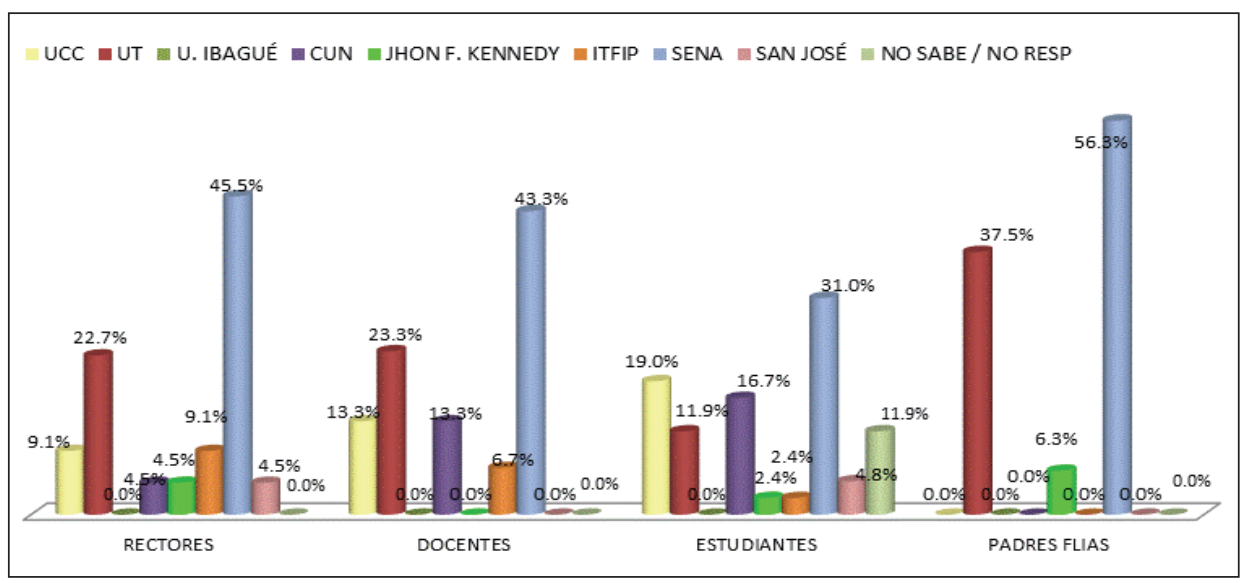

Fuente: elaboración propia.

Cabe destacar que el SENA en Colombia es la institución que abandera los procesos de articulación con la educación media; este proceso nace con la expedición de la (Ley 55 de 1985) con la cual se reordenan las finanzas del Estado. En virtud a esta normatividad, el SENA firmó un convenio con el Ministerio de Educación Nacional para mejorar los programas de educación técnica; este convenio dio lugar a la promulgación de la (Ley 119 de 1994), mediante la cual el SENA formaliza las asesorías con las instituciones de educación media-técnica, y facilita seguidamente el proceso de articulación con éstas. 
La expedición de la (Ley 749 de 2002), "por la cual se organiza el servicio público de la educación superior en las modalidades de formación técnica profesional y tecnológica”, abre la posibilidad de articulación de la educación media con la educación superior.

\section{Categoría: Modificación del proyecto educativo institucional - PEI-, producto de la articulación}

En la figura número 3 , se visualiza que los rectores manifiestan haber modificado el PEI con el proceso de articulación, no obstante, al hacer la revisión documental en los colegios se encontró que un $90 \%$ de las instituciones no había modificado este plan; el $10 \%$ restante de las instituciones se encuentran en proceso de ajuste de este documento, el cual las proveerá de una mejor actuación en el sector educacional. Un resultado significativo en este aspecto, lo representa el $2.8 \%$ arrojado por los docentes al preguntárseles si la articulación había generado alguna modificación en el proyecto educativo institucional.

Figura 3

Modificación del proyecto educativo institucional -PEl-, producto de la articulación.

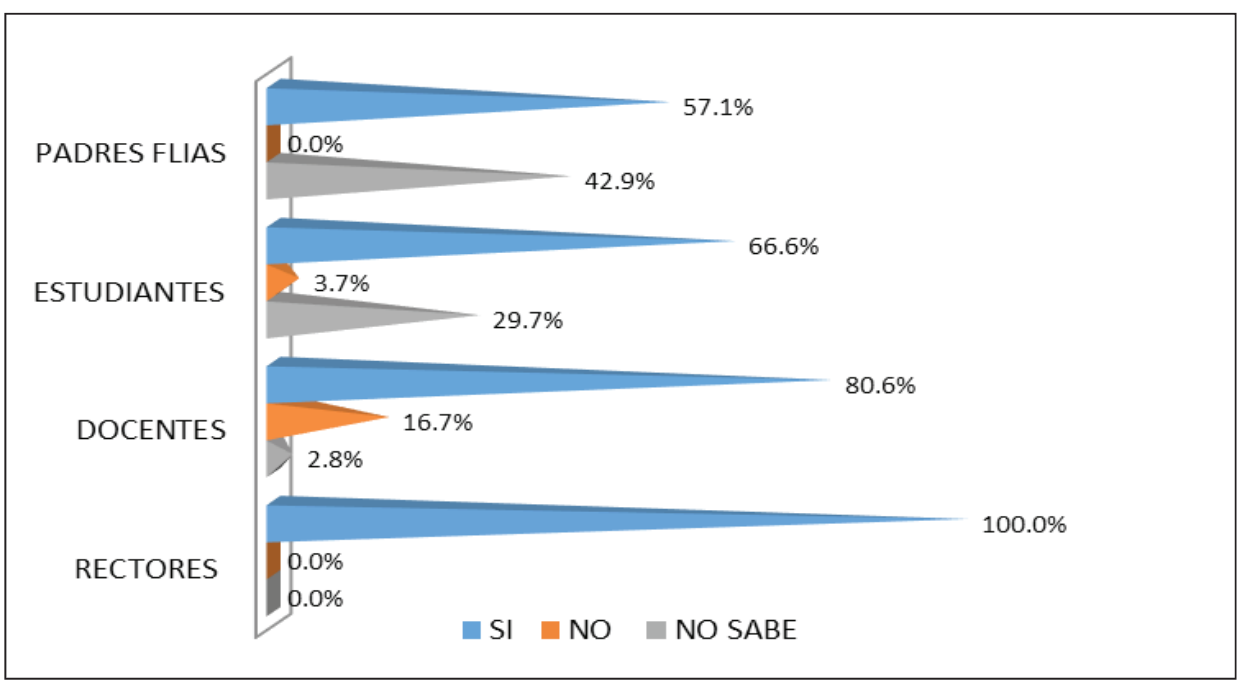

Fuente: elaboración propia.

El artículo 73 de la (ley 115 de 1994), establece que en Colombia "cada establecimiento educativo deberá elaborar y poner en práctica un Proyecto Educativo Institucional (PEI), con el fin de lograr la formación integral del educando". En este documento, entre otros, debe estar contemplado el proceso de articulación, considerándose que la pretensión es alcanzar estándares de calidad educativa, la cual responda a las necesidades del sector productivo del país. Así se ha entendido por parte de los establecimientos educativos estudiados, quienes han modificado dicho proyecto al incluir el proceso de articulación con fines de orientar la institución hacia el logro de sus objetivos. 


\section{Categoría: Preparación del personal docente para llevar a cabo la articulación}

Sobre la calidad y preparación de los docentes inmersos en el proceso de articulación, la figura número 4 indica que el 93.3\% de los rectores respondió que existen los docentes calificados para llevar a cabo el proceso de articulación; en constraste con el porcentaje arrojado por los padres de familia $(100 \%)$, se puede interpretar como un resultado contradictorio, debido a que los rectores suponen tener más conocimiento sobre sus profesores en cuanto la prepración para desarrollar este tipo de procesos.

\section{Figura 4}

Preparación del personal docente para llevar a cabo la articulación

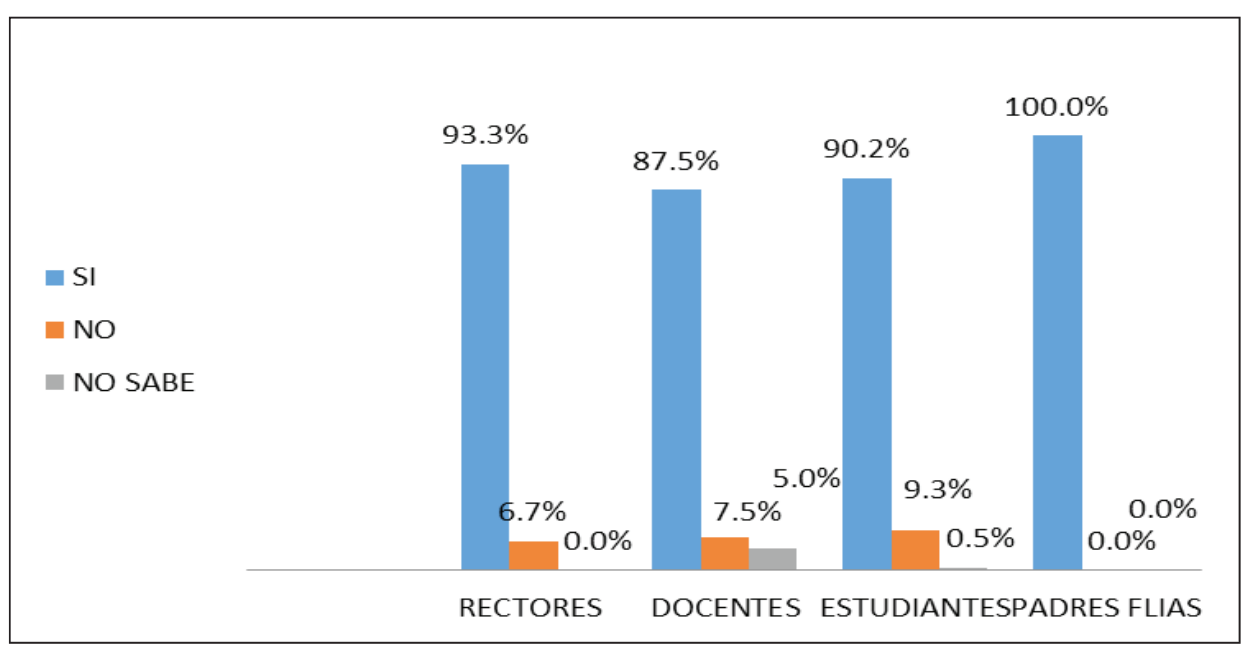

Fuente: elaboración propia.

Las instituciones educativas articuladas con el SENA se fevorecen, en razón a que esta entidad brinda capacitación a los docentes de grado $10^{\circ} \mathrm{y}$ $11^{\circ}$ que se encuentran en el proceso de articulación; es de anotar, que en algunos casos los docentes que se hallan en este proceso no tienen el perfil requerido para llevar a cabo la articulación. Asimismo, se destaca que en la articulación que se adelanta con las universidades, no existen capacitaciones dirigidas a los docentes de las escuelas de educación media.

\section{Categoría: Calidad en la enseñanza-aprendizaje luego de llevar a cabo la articulación}

Al preguntárseles a los rectores acerca de la articulación como factor influyente en los procesos de enseñanza-aprendizaje, según la figura número 5, el $93 \%$ respondió que "sí" se mejora la calidad de la enseñanza con la implementación de la articulación y un $7 \%$ respondió que "no sabía". Parece ilógica y a la vez irracional esta respuesta exteriorizada por parte de la minoria de los rectores ecuestados, en razón a que el organo maximo 
de dirección de una institución educativa funcionalmente, no conozca la importancia que representa para la comunidad educativa un proceso de articulación.

\section{Figura 5}

Calidad en la enseñanza-aprendizaje luego de llevar a cabo la articulación
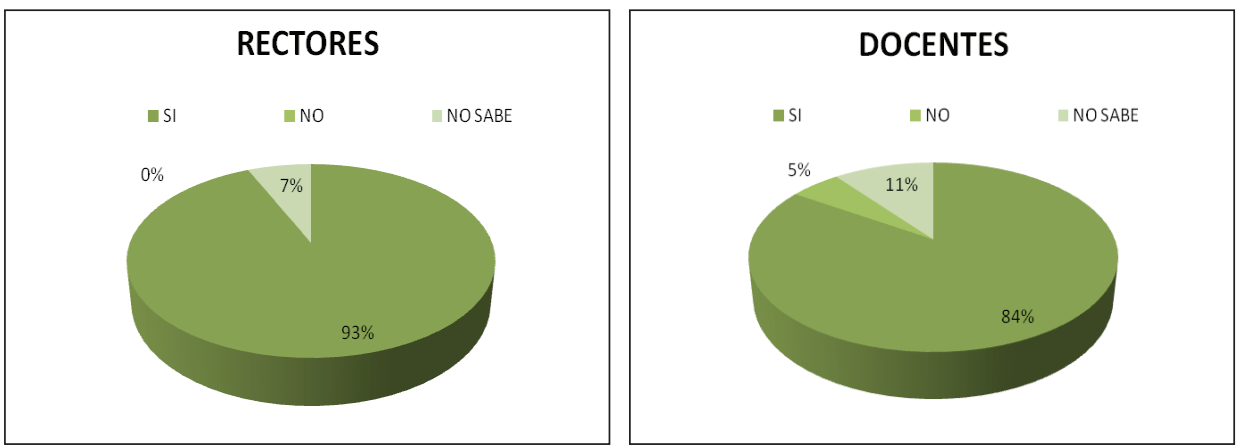

Fuente: elaboración propia.

Uno de los principios que orienta la articulación consiste en "lograr altos niveles de desempeño en las competencias básicas, ciudadanas y específicas de los estudiantes, brindando oportunidades de exploración y profundización en las distintas áreas del conocimiento y asegurando que los procesos educativos y administrativos se gestionen con criterios y estándares de calidad" (MEN-SENA, 2012).

\section{Categoría: Disminución de la deserción escolar a causa de la articulación}

En la figura número 6 se observa que los rectores sostienen en un $67 \%$ que la articulación "si" ha disminuido la deserción escolar, un 20\% manifiesta que "no" y un $13 \%$ "no sabe". El resultado evidencia en alto grado porcentual que efectivamente ha disminuido la deserción escolar, pero no es propiamente por causa de la articulación, sino, por los programas que está utilizando la Secretaría de Educación Municipal como instrumento para reducir los índices de deserción; entre otros, el desayuno escolar, la gratuidad en la educación y subsidios a los uniformes y transporte escolar. Todos estos beneficios se han implementado con el fin de retener a los estudiantes en las escuelas.

A pesar de los ingentes esfuerzos que concentra el Gobierno Nacional por aumentar la cobertura y la calidad educativa, existen múltiples causas que están originando la deserción en la educación en Colombia; entre otras, se puede desatacar: la violencia escolar, conflictos entre directivos, docentes y estudiantes, maltrato entre compañeros, considerar la educación cómo poco útil, poca motivación para estudiar, pedagogías inadecuadas, etcétera. De igual forma, no se puede desconocer que los procesos de articulación mejoran la permanencia de los alumnos en el proceso educativo y disminuyen la deserción escolar (MEN-SENA, 2012). 


\section{Figura 6}

Disminución de la deserción escolar a causa de la articulación
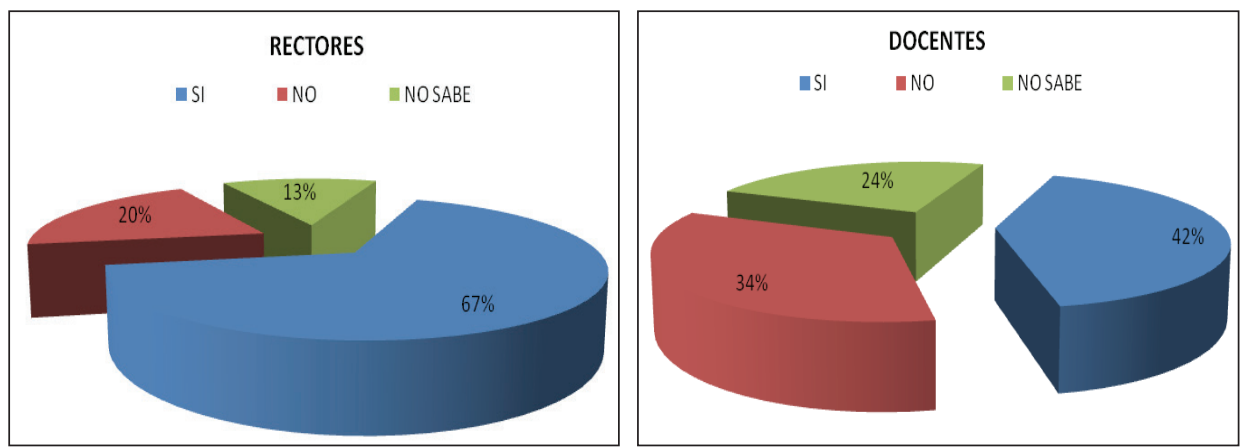

Fuente: elaboración propia.

\section{CONCLUSIÓN}

Los entes gubernamentales deben orientar esfuerzos en búsqueda de generar una articulación efectiva entre las instituciones, el sector productivo y la sociedad; lo anterior, debe implicar el rediseño de los proyectos educativos institucionales -PEI-, con el fin de encausarlos hacia la calidad educativa, las competencias laborales y evitar la deserción estudiantil. En la actualidad se refleja una ausencia de políticas y estrategias que se emitan desde el orden municipal, que puedan fortalecer estos ejes y a su vez conducir a disminuir la tasa de desempleo en la ciudad.

El objetivo del proceso de articulación de la educación media con la educación superior, "consiste en disminuir la pobreza con mayor empleo para mejorar la productividad regional” (MEN-SENA, 2012, p.7). No obstante, en el municipio de Ibagué no existe un estudio que permita identificar si la articulacion ha justificado la vinculacion con el sector productivo. La desconexión existente entre el proceso de articulación y el medio empresarial, ha generado en la inserción laboral un impacto negativo; en casos muy excepcionales por la magnanimidad de las empresas, ubican a los estudiantes para que lleven a cabo su práctica empresarial, logrando vincularse algunos de ellos laboralmente de forma indefinida.

Las instituciones de educación media articuladas con el SENA brindan capacitación a los docentes que orientan los cursos de los grados $10^{\circ}$ y $11^{\circ}$, y que se encuentran en el proceso de articulación. Cabe destacar que en algunos casos, los docentes que se hallan en esta etapa transitiva no tienen las suficientes competencias y el perfil requerido para llevar a cabo dicho proceso, por tal razón demandan una formación que les permita cumplir cabalmente con el efecto esperado. Por su parte, las universidades que llevan a cabo la articulación, no precisan programas de capacitación dirigidos a los docentes de las escuelas de educación media en este ámbito.

Las competencias básicas para el desempeño laboral las define el sector productivo; es decir, son las empresas las que de una u otra manera deben 
conferir los parámetros para determinar la mano de obra calificada que demanadan y en que áreas. Infortunadamente, las instituciones educativas del municipio de Ibagué no tienen en cuenta al sector productivo para identificar estas competencias en el proceso de articulación. Existe una real desconexión entre los requerimientos del sector productivo y/o económico, y los procesos de articulación que llevan a cabo las instituciones de educación media; aunque varias de éstas manifiestan que adelantaron estudios relacionados con este tema en su momento, dichos estudios no cuentan con una evidencia física.

La participación de la Secretaría de Educación para con las instituciones educativas está dada con la firma de algunos convenios, y con el favorecimiento a los rectores en el nombramiento de docentes capacitados para el proceso de articulacion, cuando se cuenta con el presupuesto destinado para atender este fin. El municipio de Ibagué en cabeza de la Secretaría de Educación, no realiza un seguimiento al proceso de articulación que se adelanta en las instituciones de educación, y tampoco está al tanto de los convenios que éstas instituciones firman con otros órganos educativos. Los docentes son consultados y sirven de apoyo en el proceso de articulación de la educación, sin embargo, el grupo de docentes que conoce y administra estos procesos en las instituciones educativas es muy reducido.

Finalmente, de todo lo anterior se puede colegir que es necesario identificar todos aquellos aspectos que componen el proceso articulativo en Colombia, a fin de implementar una estrategia cuyo impacto genere una verdadera revolución en el sector educativo. No es novedoso encontrar instituciones de educación superior que hayan implantado un modelo institucional de educación media, técnica y tecnológica, pero sí lo puede llegar a hacer, cuando se establezca una supervisión en la cual se verifique por parte de dichas instituciones, si las prácticas adelantadas en la educación media tienen correspondencia con la exigencia y la calidad requerida por éstas.

\section{REFERENCIAS BIBLIOGRÁFICAS}

Burbules, N. \& Torres, C. (2001). Globalización y educación. Ministerio de Educación Cultura y

Deporte de España. Revista de educación, No. extraordinario.

Camargo, E., Garzón, E. Y., \& Urrego, L. S. (2012). Articulación de la educación media y superior para Bogotá. Visión electrónica, 6(2), 160-171.

Díaz, A. (Marzo, 2012). Sobre tres aspectos en el porvenir de nuestra educación superior.

Contribución de la Universidad del Rosario al debate sobre Educación Superior en Colombia. Ponencias presentadas por los miembros de la Comunidad Rosarista dentro del Ciclo de Foros sobre Educación Superior, Bogotá, Colombia.

Diaz, C. (2012). La política de articulación entre la educación media y la superior. El caso de los programas de la Secretaría de Educación de Bogotá. Investigación \& Desarrollo, 20(2).

Guerrero-Mosquera, A.; Martínez-Benavides, J. A. \& Guazmayán-Ruiz, C. A. (2012). Articulación entre la educación media y superior: Universidad de Nariño. magis, Revista Internacional de Investigación en Educación, 4 (9), 741-753.

Hernández, R., Fernández, C. \& Baptista, M. (2010). Metodología de la Investigación. México: Mc Graw Hill.

Ley 55. Por medio de la cual se dictan normas tendientes al ordenamiento de las finanzas del Estado y se dictan otras disposiciones (1985).

Ley 115. Por la cual se expide la ley general de educación (1994).

Ley 119. Por la cual se reestructura el Servicio Nacional de Aprendizaje, SENA, se deroga el Decreto 2149 de 1992 y se dictan otras disposiciones (1994).

Ley 749. Por la cual se organiza el servicio público de la educación superior en las modalidades de formación técnica profesional y tecnológica (2002).

Ministerio de Educación Nacional. (2008). Articulación de la educación con el mundo productivo.

Competencias Laborales Generales. Recuperado el 17 de abril de 2015, de http://www.mineducacion.gov. co/1621/articles-106706_archivo_pdf.pdf

Ministerio de Educación Nacional (2009). Orientaciones para la articulación de la educación media. Bogotá, Colombia.

Ministerio de Educación Nacional (2010). Lineamientos para la articulación de la educación media. Bogotá, Colombia. 
Ministerio de Educación Nacional \& Servicio Nacional de Aprendizaje SENA. (2012). Articulación de la educación media con la educación superior, la formación profesional integral y la educación para el trabajo y el desarrollo humano. Bogotá, Colombia.

Ministerio de Educación Nacional (2012). Caracterización de procesos de articulación. Bogotá, Colombia.

Misas, G. (2004). La educación superior en Colombia: análisis y estrategias para su desarrollo. Bogotá, Colombia: Universidad Nacional de Colombia (p.298).

Proyecto principal de educación para América Latina y el Caribe. PROMEDLAC VI \& MINEDLAC VII (1996). Recomendación de Kingston: Educación, Democracia, Paz y Desarrollo. Recuperado el 18 de abril de 2015, de: http://unesdoc.unesco.org/ images/0010/001047/104761s.pdf
Schwartzman, S. (2001). El futuro de la educación en América Latina y el Caribe. Oficina Regional de Educación de la UNESCO para América Latina y el Caribe, UNESCO-Santiago.

Uzuriaga, V., Posso, A. \& Martinez, A. (2013). Algunas estrategias para mejorar la articulación de la educación media con la superior. Scientia et Technica, 18(4), 732-736.

Vasco, C. (2006). Siete retos de la educación Colombiana para el período de 2006 a 2019. Medellín (Antioquia), Colombia, presentada como conferencia por la Universidad EAFIT de Medellín. Vela, J. (2000). Educación superior: Inversión para el Futuro. Revista Cubana de Educación Superior, 20(1), 3-16.

Weiss, E. (2012). La educación media superior en México ante el reto de su universalización. Archivos de Ciencias de la Educación, 6(6), 1-22. 